\title{
Guidelines of the Indian Society for Sleep Research (ISSR) for Practice of Sleep Medicine during COVID-19
}

\author{
Ravi Gupta ${ }^{1} \cdot$ V. Mohan Kumar ${ }^{2} \cdot$ Manjari Tripathi $^{3} \cdot$ Karuna Datta $^{4} \cdot$ Manjunatha Narayana $^{5}$. \\ Kripesh Ranjan Sarmah ${ }^{6} \cdot$ Manvir Bhatia $^{7} \cdot$ Preeti Devnani $^{8} \cdot$ Sourav Das $^{9} \cdot$ Deepak Shrivastava $^{10}$. \\ Rama Devi Gourineni ${ }^{11,12} \cdot$ Tripat Deep Singh $^{13} \cdot$ Apar Jindal $^{14} \cdot$ Hruda Nanda Mallick $^{2}$
}

Published online: 4 July 2020

(c) Springer Nature Singapore Pte Ltd. 2020

\begin{abstract}
Background Sleep services are assigned a non-essential status during COVID-19. The American Academy of Sleep Medicine strongly urges sleep clinicians to continue postponing non-urgent care until a later date, if such a recommendation is made by state officials due to local conditions. At the same time, one cannot ignore the fact that sleep is important for people's health and wellbeing. Therefore, to protect the health of the population, it is essential to find ways and means to continue the practice of sleep medicine even during the COVID-19 pandemic.

Method Social environment and work ethics in sleep clinics and sleep laboratories in Asia, Africa, and Latin America are different from those in the US. Under these circumstances, the Indian Society for Sleep Research (ISSR) created a task force to develop guidelines for the practice of sleep medicine, not only for the Indian environment but also for other countries that are affected by the COVID-19 pandemic. The task force examined documents regarding practice of sleep medicine and associated specialities during COVID-19 by various professional organizations and governmental authorities. The recommendations were examined for their applicability. Wherever gaps were identified, consensus was reached keeping in view the available evidences.

Outcome and Recommendations The emphasis of the guidelines is on avoiding doctor to patient contact during the pandemic. Teleconsultation and other modes of audio-visuals can be used as modes for medical practice during the COVID-19 pandemic. However, in addition to the patient, the presence of a family member, or a reliable informant is recommended. Patients of most sleep disorders can be provided tele-aftercare service. ISSR guidelines also give a list of medications allowed to be prescribed during the first and the follow-up teleconsultation. Hospitals and clinics are slowly opening in India and many other countries. As sleep services resume operations, there is a need to find innovative ways to reduce contact with COVID-19 patients, follow personal protection guidelines, as well as social distancing. This article does discuss strategies for the safe conduct of Level 1 sleep studies. Home sleep testing, which had greater acceptance during the last few years, should be given more attention during the COVID-19 period. Once the decision to reopen the sleep laboratory and resume operations is made, the safety of the patients and office staff should become the major priority. The ISSR recommendation is to postpone and reschedule in-laboratory positive pressure therapy, but it mentions the considerations to be followed in emergency situations. At the same time, high clinical risk patients may be diagnosed on the basis of clinical findings, and without performing polysomnography or home sleep testing. However, at some point, there is a need to reinitiate the in-lab testing. In addition, daily assessment of the COVID-19 situation in the community, along with a review of the situation with local public health and the state health department is advised.
\end{abstract}

Keywords Teleconsultation · COVID-19 · Pandemic · Polysomnography $\cdot$ In-lab testing $\cdot$ Level-1 sleep study $\cdot$ Sleep medicine $\cdot$ Home sleep study $\cdot$ Personal protective equipment (PPE) · Positive airway pressure (PAP) $\cdot$ Titration

V. Mohan Kumar and Hruda Nanda Mallick: Formerly at Department of Physiology, All India Institute of Medical Sciences, New Delhi 110029, India.

Extended author information available on the last page of the article 


\section{Background}

All hospitals, and medical practice itself, are grossly affected throughout the world during the novel coronavirus (COVID19) pandemic. The practice of sleep medicine is probably more affected than the other medical disciplines. Therefore, it needs to be modified and adapted to the prevailing pandemic situation. Indian Society for Sleep Research (ISSR) created a task force to develop guidelines for the practice of sleep medicine that are applicable not only to India but also to other countries that are affected by the COVID-19 pandemic.

\section{Methods}

To achieve the goal, ISSR identified scientists who are actively engaged in the practice of sleep medicine and have sufficient experience in running sleep clinics and sleep laboratories. Members were requested to examine guidelines proposed by various professional sleep societies as well as related specialities. In addition, the members also examined guidelines proposed by governmental authorities for general medical practices. Responsibilities of examining specific questions as depicted in Subsects. 4.1-4.3 of the present document was divided among members. The initial documents of respective sections prepared by the members were circulated. An online meeting was conducted and conclusion was drawn after discussing available scientific evidences. Thus, the telemedicine section of the present guidelines is specifically based upon the recommendations from Indian authorities and other countries may adapt it according to the prevailing local norms.

\section{Outcome}

The American Academy of Sleep Medicine (AASM) has issued mitigation strategies to help sleep medicine clinicians in their professional activities during the spread of COVID-19 [1]. Their recommendations are based on the recommendations of Centres for Disease Control and Prevention to contain and avert infection of COVID-19 in health-care settings [2]. European Sleep Research Society has published guidelines on how to manage sleep during Covid-19, especially pertaining to Cognitive Behavioural Therapy for Insomnia [3]. The British Sleep Society has made statements on sleep-related advice to patients during the Covid-19 pandemic [4]. The American Clinical Neurophysiology Society guidelines describe in detail about technologist safety and staffing, equipment maintenance and cleaning, managing requests for neurodiagnostic testing, and physician staffing [5].

Of all these available online advisories, AASM guidelines stand out not only because they deal with sleep clinic and sleep center strategies in detail, but they also address every aspect related to the COVID-19 pandemic in the US [1]. Social environment and work ethics in sleep clinics and sleep laboratories in Asia, Africa, and Latin America are different from those in the US. Though the novel coronavirus does not choose its victim on the basis of nationality or regions, there are certain social distancing norms in different countries which may or may not be conducive to the spread of COVID-19 through contact. Moreover, in India and in many other countries, sleep clinics and laboratories are, by and large, part of major hospitals. The hospitals in many countries (even expensive private hospitals) are generally overcrowded. The hospital culture, generally practiced in these countries, is also conducive to the spread of any pandemic. Taking into account all these facts, the Government of India has issued guidelines to prevent the spread of COVID-19 infection in the workplace [6].

Guidelines issued by the government on preventive measures to contain the spread of COVID-19 in workplace settings are also applicable to hospitals [6]. Indian Medical Association's Hospital Board of India has released basic guidelines for the doctors and hospitals of the country [7]. These guidelines address strategies to prevent infection in various areas of hospitals. For example, their guidelines for hospital reception are aimed not only to protect people working in reception areas but also to identify suspected COVID-19 cases right at the entry point itself [7]. While Out-Patient Department guidelines are applicable to sleep medicine clinicians, guidelines for the In-Patient Department and hospital staff are applicable to the sleep laboratory and its staff [7]. Similarly, attendants' guidelines are something very much applicable to those people accompanying the patients coming for sleep studies [7].

India is in the forefront of combating the COVID-19 pandemic, like other countries around the world. Although sleep medicine is not considered an essential service, the lack of adequate sleep can reduce immunity, and the ability to handle stress, and organ dysfunctions that occur during this pandemic [8]. Sleep disorders such as obstructive sleep apnea (OSA) and insomnia continue to be prevalent in the Indian population $[9,10]$. OSA contributes to cardiovascular, metabolic, neuro-cognitive, social, occupational, and traffic complications. In fact, OSA with cardiovascular complications may be a collateral risk factor for COVID-19 disease [11]. Thus one cannot ignore the fact that sleep is important for people's health and wellbeing. Therefore, to protect the health of the population, it is essential to find ways and means to continue the practice of sleep medicine even during the COVID-19 pandemic. 
All the guidelines lay emphasis on avoiding doctor to patient contact during any pandemic. This is certainly an important consideration during the practice of sleep medicine. Home sleep testing, which had greater acceptance during the last few years, is gaining more attention during the COVID-19 period. Various levels of sleep studies, telemedicine, and positive airway pressure (PAP) therapy are the major aspects, given emphasis by the task force group of ISSR in these guidelines.

\section{RECOMMENDATIONS}

The Task Force made following recommendations:

\subsection{Consultation Through Telemedicine}

Teleconsultation is one important mode suggested for medical practice during the COVID-19 pandemic. Ministry of Health and Family Welfare, Government of India has issued guidelines for using teleconsultations during medical practice [12]. These guidelines are important for the practice of sleep medicine, not only in India but also in other countries and were published in the Gazette of India notification dated 14 May 2020. They have clearly defined the rules to be followed during the first teleconsultation and the followup teleconsultations [13]. Discrimination between the two is important as modes of consultation and the medications that may be prescribed differ between the two categories.

\subsubsection{Definitions of First and Follow-Up Consultations [12]:}

These guidelines define the first consultation as meeting any of the following criteria:

- patient consulting the physician for the first time

- not consulted the same physician at least 6 months prior to the present consultation

- has consulted the same physician within the past 6 months, but the physician is not able to recall advice given on a previous consultation

- the symptoms for which the present consultation is sought are unrelated to disease spectrum of the previous consultation or not arising out of treatment related to the previous consultation

On the other hand, a follow-up consultation is defined as:

- consultation with the same specialist within six months of the previous consultation for the conditions arising out of disease spectrum of treatment of disease for which the first consultation was sought [12]

\subsubsection{Selecting Patients for Telemedicine Consultation}

Almost all patients can be followed up in telemedicine through different available modes. However, many patients with sleep disorders may not be aware of their symptoms as they occur during sleep, especially those suffering from sleep apnea, parasomnia, and hypersomnia. Hence, in addition to the patient, the presence of a family member or another reliable informant is preferred during teleconsultation for sleep disorders. For patients, where physical examination is required for making a decision, consultation should not be continued until a primary care physician is available with the patient. The specialist is not bound to provide teleconsultation unless the desired information and investigations are available to him. In such situations, the patient may be advised to seek an opinion from a nearby physician [12].

The use of teleservices in sleep medicine has increased in internet-based cognitive behavior therapy and telemonitoring of PAP devices, with promising results $[14,15]$. Results of teleconsultation have been found to be comparable to face-to-face interaction for cognitive behavior therapy for insomnia, training of PAP and mask fitting, initiation of therapy after in-lab titration, and follow-up consultations for PAP therapy [14-17]. Considering these facts, patients of most sleep disorders can be managed through teleconsultation. However, patients requiring invasive or surgical procedures for the diagnosis or management of sleep disorders, cannot be helped through teleconsultation [12].

\subsubsection{Responsibility of the Platform Providing Teleconsultation}

The telemedicine technology platform should display the contact information and identity of the practitioner and this is the responsibility of the service provider [12]. Therefore, the credentials of the practitioners have to be verified and evaluated before enrolling them in the telemedicine platform [12]. The telemedicine technology provider needs to ensure a mechanism where queries or grievances of the customer can be placed [12].

\subsubsection{Reliability of Information Provided by Patient and Informant}

For many sleep disorders, diagnosis and follow-up are dependent upon clinical information provided by the patient and their family members. Thus, information is subjective and qualitative. Many of the sleep disorders e.g., sleep apnea, parasomnia, and hypersomnia may have associated medico-legal issues [18-21]. Hence, the sleep physician should ensure the identity of the patient before providing consultation [12]. 
4.1.4.1 Subjective and Qualitative Data In conditions where diagnosis and intervention are based on subjective and qualitative information viz., the occurrence of symptoms or improvement in clinical symptoms, change in functioning measured by activities of daily living or quality of life, teleconsultation should preferably be done in the presence of another person, as mentioned earlier. In addition, the use of questionnaires may also assist in converting subjective and qualitative data into semi-quantitative data, and is encouraged during the practice of sleep medicine.

4.1.4.2 Objective and Quantitative Data Objective and quantitative data can be obtained through sleep diary, actigraphy, and PAP devices. The data obtained from the latter two modes can be considered as reliable. However, data from the sleep diary should be considered in the background of clinical assessment.

\subsubsection{Choosing Platforms for Teleconsultation}

Technical specifications of the equipment, viz. bandwidth, resolution, etc. are provided in a position paper of AASM $[16,17]$. Hence, the task force of ISSR is not touching those aspects. Two different modes are available for teleconsultation $[12,17]$.

4.1.5.1 Synchronous Mode Synchronous teleconsultation allows practitioners to serve patients in real-time $[8,12]$. This mode is similar to regular face-to-face consultation in many aspects and allows both parties to explore other issues that are relevant and important for diagnosis and management. The synchronous mode can be audio-video consultation (e.g., Skype, Zoom, Google Hangout, WhatsApp etc.), or audio-consultation using mobile and landline. Some professional bodies do not consider the latter mode of consultation as teleconsultation [12]. Texting and chatting through short message service (SMS) also come in this category.

4.1.5.2 Asynchronous Mode During asynchronous teleconsultation, patients send their information or query to the physician through e-mail, WhatsApp, or SMS, and the physician responds to them at his convenience [12]. This mode provides the flexibility of working to both parties. In addition, through some of these media, patients may share relevant videos of symptoms, previous or present prescriptions, lab reports, sleep diaries, adherence diaries which help the physician in making better decisions. However, this mode is not good for situations requiring prompt attention.

Asynchronous modes may also be used for awareness programs as physicians may use educational videos, short films, or interactive voice response systems to help patients with a specific condition [12].
4.1.5.3 Choosing the Modes ISSR guidelines recommend the use of audio-video-based consultation during the first consultation. However, during follow-up consultation, any of the modes described above may be used [12].

\subsubsection{Transmission of Data to Sleep Specialist}

Specialists should encourage patients to share semi-objective data like sleep diary or reports of other investigations. This facility is available with almost all the modes described above, except SMS. Having complete information helps in a thorough assessment of medical conditions and increases the accuracy of diagnosis as well as planned intervention.

However, gathering data from some of the devices used in sleep medicine practice e.g., portable-polysomnography, actigraphy and PAP machines may require docking stations or specific software to download the data and prepare reports. Without a docking station and software, data from these devices cannot be transmitted to a physician for assessment and decision making. Fortunately, certain new models of PAP machines have the facility of wireless data transmission which can be made available by the patient, either to the service provider or to the laboratory. This data is reliable and can be effectively used for the initiation of PAP therapy, counseling of patients to improve adherence as well as for follow-up of patients [15].

\subsubsection{Documentation and Record-Keeping}

Documents should be prepared and the record should be kept the way it is done in face-to-face consultation [12]. Hence, the minimum record should be maintained including history, examination (that can be performed through teleconsultation), investigations, and prescriptions [12, 22]. In addition, call logs should be maintained. Record of prescription should be kept either in a physical or in an electronic form $[12,22]$.

\subsubsection{Precautions and Safety Measures}

- Ethical issues should be diligently dealt with as done during face-to-face practice.

- Every step should be taken to ensure the confidentiality of the patient's data at the physician's end. This can be done by arranging password-protected meetings for video-consultation and ensuring the protection of passwords for mails.

- Explicit consent of the patient is required for recording the video-consultation.

- The patient should not be forced for teleconsultation if he wants to pay an in-person visit to the physician's office. 
Table 1 Medications allowed to be prescribed during first and follow-up teleconsultation [12, 22]

\begin{tabular}{|c|c|c|c|c|}
\hline Category & Representative medications & First consultation & Follow-up consultation & Remarks \\
\hline $\mathrm{O}$ & $\begin{array}{l}\text { Pain-killers, ORS, Vitamins, } \\
\text { food supplements, anti- } \\
\text { allergic, lozenges, antacids, } \\
\text { laxatives }\end{array}$ & $\begin{array}{l}\text { Over the counter, no prescrip- } \\
\text { tion required }\end{array}$ & $\begin{array}{l}\text { Over the counter, no prescrip- } \\
\text { tion required }\end{array}$ & \\
\hline A & $\begin{array}{l}\text { Antidepressants: Imipramine, } \\
\text { Fluoxetine, Escitalopram } \\
\text { Antiepileptics: Phenobarbi- } \\
\text { tone, Phenytoin, Carbamaz- } \\
\text { epine, Valproate Hypnotics: } \\
\text { Clonazepam and clobazam } \\
\text { Antipsychotics: Haloperidol, } \\
\text { Risperidone, Olanzapine }\end{array}$ & $\begin{array}{l}\text { Prescribe through Video Con- } \\
\text { sultation only }\end{array}$ & $\begin{array}{l}\text { Prescribe using any mode of } \\
\text { consultation }\end{array}$ & $\begin{array}{l}\text { Do not prescribe Zolpidem and } \\
\text { Lorazepam during telecon- } \\
\text { sultation }\end{array}$ \\
\hline B & $\begin{array}{l}\text { All other antidepressants, } \\
\text { antipsychotics, antiepileptics, } \\
\text { pregabalin, drugs for demen- } \\
\text { tia, Anti-craving medications }\end{array}$ & Can't be prescribed & Can't be prescribed & \\
\hline
\end{tabular}

\subsubsection{Legal Issues}

- Advertisements to solicit patients cannot be made on any platform [12].

- Consent is considered as implied when a patient is consulting the physician.

- In the absence of a health-care provider at the patient's end, consent should be taken from the patient that he/ she understands the limitations of providing professional advice without physical examination [22].

\subsubsection{Limitations of Teleconsultation in Sleep Medicine}

- Patients with hypersomnia that require repeat Multiple Sleep Latency Test (MSLT) or Maintenance of Wakefulness Test (MWT) cannot be helped through telemedicine.

- Settings of PAP devices, if required, are difficult to be changed through teleconsultation.

- Fitting issues of interface with PAP devices, though possible, nevertheless are difficult to be resolved, using teleconsultation.

- Other laboratory investigations may be difficult to get done in a COVID-19 pandemic situation.

\subsubsection{Medical Prescription}

Patients should be provided a copy of the medical prescription, through asynchronous modes after scanning or generating it electronically $[12,22]$. Neuro-psychotropic medications are often used for the treatment of sleep disorders. They should be prescribed following the guidelines for medical practice in India [12, 22]. There are some restrictions on their use in telemedicine practice (Table 1).

\subsection{Guidelines for Conducting Sleep Study}

Ministry of Health and Family Welfare, Government of India is regularly issuing advisories regarding the community status of COVID-19 spread, lockdown orders for public safety. They should be taken into account while reopening the sleep laboratories in India. A designated staff member should be made responsible for regular monitoring of COVID-19 updates from state and local health departments. Sleep clinicians should be prepared to adjust operations as local conditions change. Short-term closure of the sleep center may be needed in response to the surge in local community transmission. Sleep studies during the COVID-19 pandemic may be conducted in the laboratory or at home, depending on the level of the community spread and the condition of the sleep disorder.

\subsubsection{Magnitude of COVID-19 Transmission in Community and Decision of Sleep Study}

A. During large scale community transmission of COVID19 , with the prevalence of multiple cases within the community, the ISSR recommends to postpone and reschedule in-laboratory as well as home sleep tests. When sleep medicine services are postponed, communication with patients should be maintained so that their access to the medical team and continuity of care are preserved and follow-up is ensured.

B. If there is a moderate transmission of COVID-19 in the community, the present recommendations are to postpone and reschedule in-laboratory PAP therapy, as mentioned above. Visitors should be limited, but if they are necessary for patient care, they should be pre-screened just like patients before permitting them entry to the sleep center. 
C. In a situation of none to minimal community transmission of COVID-19, in-laboratory administration for diagnostic polysomnography (PSG) on children and adults as well as supervised manual PAP titration may be resumed. Visitors should be allowed as per routine sleep center policy.

\subsubsection{Screening of Staff}

- Health care personnel, sleep technologists, and office staff should be screened daily for COVID-19 symptoms viz. cough, fever, chest pain, and shortness of breath. A daily log of findings should be maintained for all staff members. They should not be allowed to work if they have any of these symptoms.

- If staff members report exposure to COVID-19 positive patient, or have worked in close proximity with a COVID-19 positive patient (within 6 feet for 15 min or more), they should not be allowed to work.

- Above mentioned people should be quarantined at home if they are asymptomatic, and in the hospital, if they are symptomatic.

- Proper guidelines should be followed based on the results of the COVID-19 testing.

\subsubsection{Screening of Patients}

- Like staff members, all patients must be screened for potential symptoms of COVID-19 prior to the procedure. This may be done through teleconsultation.

- Pre-screening of patients prior to appointment can be done via phone calls, WhatsApp or online self-assessment tools.

- If testing for COVID-19 infection is available and feasible, patients should be tested in a reasonable time frame in addition to being screened for symptoms. Investiga- tions on patients with symptoms suggestive of COVID19 symptoms should be postponed while results of COVID-19 tests are pending (Table 2).

- Presumed positive cases require two negative test results $24 \mathrm{~h}$ apart before they can be scheduled.

- Suspected positive cases require COVID- 19 testings, which must show a negative result.

- All other cases can be scheduled.

\subsubsection{Safety of Staff Members and Patients}

Once the decision to reopen the sleep laboratory and resume operations is made, the safety of the patients, any attendants, or accompanying family members, technologists, office staff, and clinical providers becomes a major priority. Sleep technologists should be given Personal Protective Equipment (PPEs) such as gloves, masks, face shields. The gown should be worn during hooking up, hooking off of the patient as well as during sterilization/ disinfection of reusable parts of equipment. Handwashing is advised before and after removing PPE. Hand sanitizer is to be used when a handwashing station is not available. Staff should maintain a distance of 6 feet $(2 \mathrm{~m})$ from each other as well as caregivers of patients. Close contact with patients cannot be avoided during sleep study but should be minimized, as far as possible. Technologists should wear gloves while touching the patient. They should change gloves before touching the computer screen or other surfaces and supplies (which may be later touched by others).

\subsubsection{Before and After Sleep Study}

Sanitization of all equipment (electrodes, belts, wires, PSG apparatus etc.) should be ensured before applying them to the patient's body. Similarly, for level 1 study, the sleep laboratory should be sanitized and bed-linen must be changed

Table 2 Screening protocol for sleep study during COVID-19

\begin{tabular}{|c|c|c|c|c|}
\hline & Symptoms & $\begin{array}{l}\text { Contact with COVID- } 19^{\text {a an/ or }} \\
\text { travel history in the past } 30 \text { days }\end{array}$ & Quarantine $^{\mathrm{b}}$ & COVID- 19 testing $\left(\mathrm{RT}\right.$ PCR) ${ }^{\mathrm{c}}$ \\
\hline Status unknown & None & None & None & Testing not done \\
\hline Presumed negative & None & None & None & Testing not done \\
\hline Self-quarantined & None & Present & $\begin{array}{l}14 \text { days at home after } \\
\text { contact or history }\end{array}$ & Testing not done \\
\hline Suspected positive & Present & May or may not be present & None & Testing not done \\
\hline Presumed positive & Present & & & Positive \\
\hline Presumed recovered & No fever in 72 hours $^{\mathrm{d}}$ & & & 2 negative test results $-24 \mathrm{~h}$ apart \\
\hline
\end{tabular}

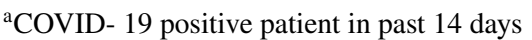

${ }^{\mathrm{b}}$ Self-quarantine for 14 days

${ }^{\mathrm{c}}$ Real time polymerized chain reaction

${ }^{\mathrm{d}}$ No fever in $72 \mathrm{~h}$ without use of antipyretics 


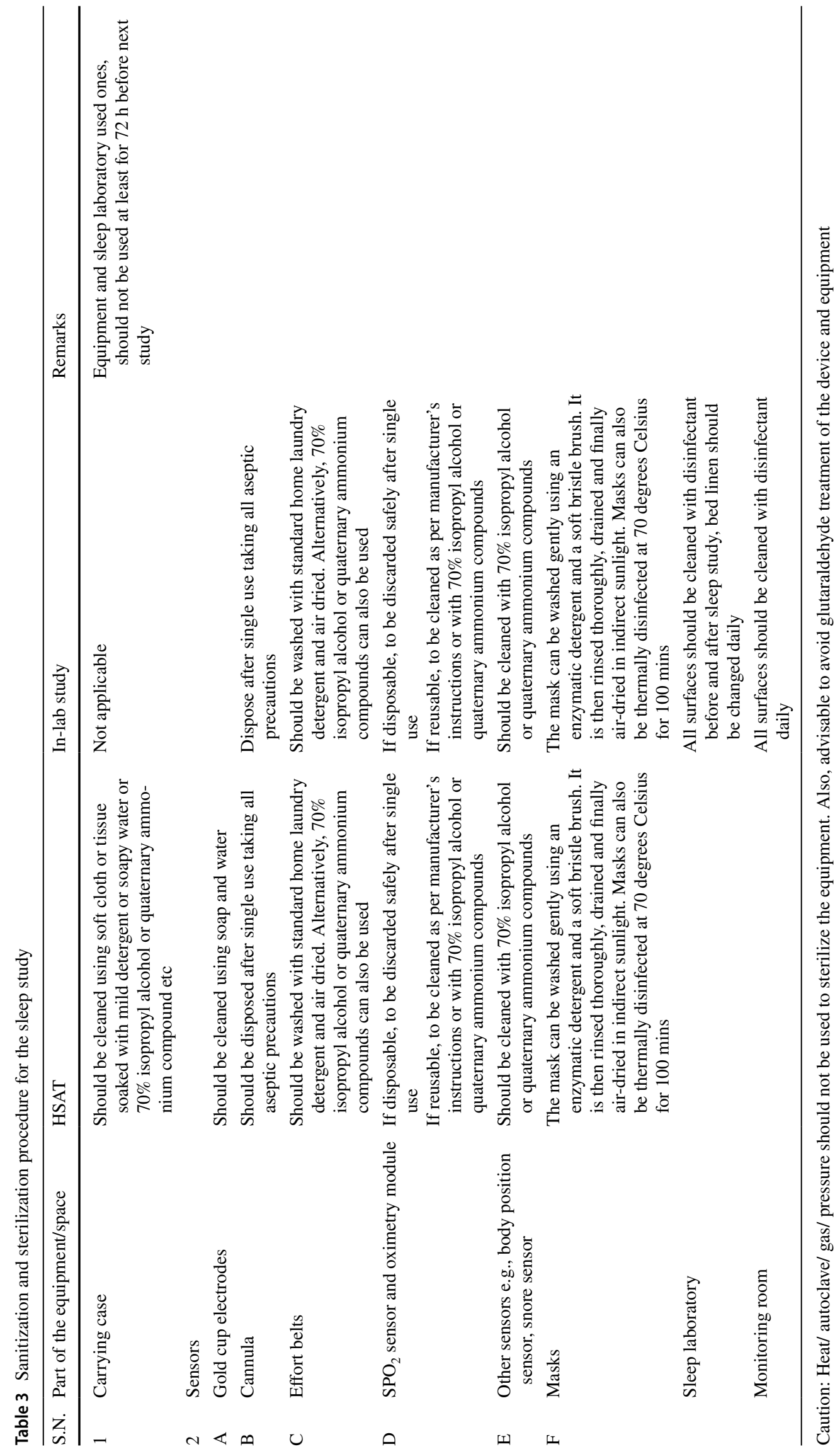


daily (Table 3). Equipment and sleep laboratory, once used, should be closed for at least $72 \mathrm{~h}$ before next use [1].

\subsubsection{Choice of Method of Sleep Study}

The sleep study can be done at home or in the laboratory. There are specific guidelines citing indications, limitations, and advantages of these methods and should be followed during COVID-19, as well [23, 24]. Till now, the Ministry of Health and Family Welfare of Government of India, has recommended postponement of all non-essential studies [25]. Like AASM guidelines, ISSR also urges sleep clinicians to postpone and reschedule sleep studies and in-laboratory administration of PAP therapy except in emergencies [1]. Further precautions during the use of these methods in the prevailing situation have been addressed by the task force of ISSR.

\subsubsection{Home Sleep Apnea Testing (HSAT)}

\section{Indications [23]}

- Home sleep apnea testing (HSAT) on adult patients should be decided by the physician, after taking into account the patient's medical history and an in-person examination.

- Home sleep testing for diagnosing OSA should be done only in medically uncomplicated adult patients with a high pre-test probability of moderate-to-severe OSA.

- Absence of associated conditions including significant cardiopulmonary disease, neuromuscular conditions, history of stroke, or chronic opioid use, central sleep disorder, such as central sleep apnea, parasomnia, narcolepsy, or severe insomnia should be ensured.

- HSAT should not be used for general screening of asymptomatic populations.

- The AASM also does not recommend the use of an HSAT for the diagnosis of an OSA in patients younger than 18 years of age [24].

\section{Disinfection of the Instrument after Sleep Study}

- Currently there is no approved official disinfectant listed by WHO or CDC for eliminating SARS-CoV2. It is likely to be considered as an emerging pathogen and therefore standard sterilization and disinfection protocols of the center should be followed.

- It is preferable to use disposable devices and/or components wherever feasible.

- Reusable device should be reused only after $72 \mathrm{~h}$ of disinfection.

- Further details may be seen in Table 3.

\section{Legal Issues}

- The Epidemic Diseases (Amendment) Ordinance, 2020 [26] has been approved by the President of India, which makes attacks on healthcare personnel or their properties (including their living and working premises) cognizable and non-bailable offenses. The offender is liable to pay compensation to the victim and twice the fair market value of the victim's property/ vehicle.

- It is also imperative for the sleep labs/employers of sleep technicians to provide health insurance coverage, at least for COVID-19, to instill confidence in their staff about their safety.

4.2.6.2 In-Lab Attended Polysomnography The practice of sleep medicine was temporarily suspended to mitigate the contagious spread of COVID-19 disease. Hospitals and clinics have been slowly opening for regular operation as India enters lockdown 5 which is otherwise stated as Unlock phase 1. As sleep services resume operations, there is a need to find innovative ways to reduce contact with COVID-19 patients, travel, follow personal protection guidelines, as well as social distancing to prevent the spread of COVID19 infection. This section will discuss strategies for the safe conduct of Level 1 sleep studies. Some studies will also require PAP titration or a daytime nap test (MSLT) the following day. In addition, pre-study and post-study consultation visits are required. However, at some point, there is a need to reinitiate the in-lab testing.

\section{Indications of Level 1 Sleep Study during COVID-19 [23,} 24]

- Patient has comorbid cardiopulmonary disease.

- Patient has history of stroke, chronic opiod use or other unstable medical conditions.

- For evaluation of complex NREM parasomnia or nocturnal epilepsy. Epilepsy monitoring is the of choice in these conditions unless co-existing sleep apnea is suspected.

- For evaluation of REM sleep behavior disorder.

- Patients with cognitive disability, who may require monitoring during the study.

- Continuous Positive Airway Pressure (CPAP) or Bi-level titration is required.

- Level 1 sleep study can be used as part of the MSLT protocol.

\section{Other Guidelines for Patient Safety}

- Waiting time should be minimized by spreading outpatient appointment times. 
- Number of patient attendants should be limited or restricted.

- Patients should be requested to maintain social distancing (6 feet away from each other in the waiting area). Indications for six feet distance on the floor should be marked and seating to be rearranged accordingly.

- All patients should be provided with masks if they do not have them.

- Hand sanitizers should be made available in multiple locations.

- Posters and other educational material stressing personal protection, handwashing, and social distancing should be placed in a sleep laboratory (keeping distance of 6 feet from others).

- Hospital premises should be frequently cleaned and sanitized, especially the frequently- touched surfaces.

- The examination couch and examination supplies should be cleaned with a sanitizer after examining each patient.

\subsection{PAP Therapy for OSA Patients during COVID-19}

All individuals with associated comorbidities like uncontrolled diabetes, hypertension, cardiac dysfunction, and underlying lung diseases are more prone to contract this infection and more likely to suffer fatality if they get infected by the SARS CoV-2 virus. The mode of spread of the virus is droplet spread all practices which involve generation of aerosols such as sneezing, coughing, talking, spitting etc. Medical device use like nebulizers and positive airway pressure (PAP) therapy devices prone individuals including, healthcare workers (HCW) and providers to infection and disease.

\section{Scope of the consensus document}

Owing to the morbidity and mortality risk amongst patients and HCWs there is a need to assess the overall risk versus benefit of using PAP therapy in patients of sleep apnea. While designing the consensus data for use of PAP devices during the COVID -19 pandemic the following general safety measures have been taken into consideration. All sleep labs are recommended to follow these measures.

a) Ensure protection of healthcare professionals including doctors, nurses, coordinators, technicians, attendants, and housekeeping staff by using recommended personal protective equipment as per the risk of exposure encountered in various settings as discussed above

b) Prevention of exposure of HCWs to infection from COVID-19 positive patients and vice versa by ensuring universal precautions, as mentioned above.

c) Prevention of cross-infection of COVID -19 amongst different patients as mentioned above.

\subsubsection{Clinically Suspected Case of OSA Without COVID-19 Diagnosis/Symptoms}

- For diagnosis of OSA, the guidelines mentioned above should be followed.

- If diagnostic testing is not possible due to high community transmission or other reasons, starting auto-CPAP therapy based on clinical suspicion alone, is still a controversial subject. A recent publication has shown that CPAP indication without a sleep study had a low sensitivity and high specificity greater than $95 \%$ in patients with high pre-test probability for OSA (snoring, report of frequent apneas, body mass index $(\mathrm{BMI})>25 \mathrm{Kg} / \mathrm{m}^{2}$ and sleepiness or tiredness plus comorbidity) [27].

- The approach suggested above is at the discretion of the treating physician.

- If the patient decides to buy the PAP device, then the delivery of auto-CPAP should be arranged. Device settings and mask fitting can be done via a video call.

- Auto-CPAP algorithm validation articles from different manufacturers should be looked into for the efficacy of the algorithm to treat OSA.

- But this approach should be limited until the pandemic continues. Once the pandemic is over, suspected OSA patients should undergo diagnostic testing first before purchasing the PAP device.

\subsubsection{Clinically Suspected Case of OSA with COVID-19 Diagnosis/Symptoms}

Wait for the PAP titration study and trial or auto-CPAP prescription until the patient recovers from the infection (assessed by RTPCR or CBNAAT negative tests).

\subsubsection{Newly-Diagnosed OSA Patient Without COVID-19 Diagnosis/Symptom}

If the patient has to go for PAP therapy to treat OSA, follow the suggestions given below:

4.3.3.1 In-Lab PAP Titration Study It can be planned in emergency situations, with the precautions mentioned in Subsect. 4.2.

If there is Large-scale community transmission, healthcare staffing significantly impacted, multiple cases within communal settings, follow the below suggestions-

a. Postpone and reschedule in-laboratory administration of positive airway pressure (PAP) therapy (i.e., PAP titration studies and split-night studies) till the time patient has been declared COVID negative by either RT-PCR or CBNAAT testing. 
b. Can plan in lab titration in emergency situations, with following considerations-

- If testing for COVID-19 infection by RT-PCR or CBNAAT is available and feasible, patients should be tested twice and declared negative before coming into the sleep center, in addition to being screened for symptoms.

- Review the potential for aerosolization and ensure technologists use appropriate Personal Protection Equipment (PPE) [28].

- Appropriate changes to air filters of air conditioning systems may need to be made in case of centralized air conditioning. It is preferable to use a separate unit for each room.

- Viral filters must be added to the PAP outlet.

- If the sleep lab is multi-bedded, they can consider allocating two fixed rooms with separate air-conditioning units for doing all in lab PAP titration. When one room is used, it should be disinfected and not used again for the next $72 \mathrm{~h}$ during which time $2^{\text {nd }}$ room can be used for inlab titration with appropriate above mentioned measures in place.

- AASM recommends that PAP administration can take place in an airborne infection isolation room (AIIR) i.e., a negative pressure room [1].

If the community transmission is none or minimal, sleep labs can resume in lab PAP titration taking above mentioned precautions.

4.3.3.2 Clinic or Home PAP Trials It can be planned in emergency situations, with the above- mentioned considerations.

If there is Large-scale community transmission, healthcare staffing is significantly impacted. Follow suggestions given below in such a situation:

a. All non-essential face to face PAP trials in the clinic or home should be avoided, to limit the potential of community spread and for the safety of staff.

b. If a patient is willing to buy the device without a trial, PAP devices can be made available to the patient, and setting up of the device can be done via a video call. An Auto CPAP will be a suitable device in this scenario if there are no clinical contraindications to it.

c. If the decision in step-b is taken, then try to follow up the patient remotely via telemonitoring/teleconferencing after $7,14,21$ and 30 days.

If the community transmission is none or minimal, sleep labs can resume in-clinic or home PAP trials with optimal precautions to ensure the safety of staff as well as the patient.

\subsubsection{Already Diagnosed OSA Patient Using PAP without COVID-19 Diagnosis/Symptoms}

OSA diagnosed patients, who are already using the PAP therapy and do not have COVID-19 symptoms or diagnosis, should continue to use the PAP therapy as before the COVID-19 situation. If the patient feels that OSA symptoms are returning during this time, then they should try to have a teleconference with their doctor to review the clinical presentation and PAP device settings.

\subsubsection{Already Diagnosed OSA Patient Using PAP with COVID-19 Diagnosis/Symptoms}

4.3.5.1 Patient in Home Quarantine It is very hard to prevent infection of other family members if one stays at home due to asymptomatic shedding of the virus, as the spread of the infection via droplets is the common mode of the virus spread. If the patient wishes to continue to use PAP at night while quarantined at home, the following advice should be given:

- Patients should sleep in a separate room, and use a separate bathroom and kitchen, if possible. Strict isolation protocol should be followed to prevent infection to family members.

- If mucus or nasal discharged seen inside the mask, he/she should be requested to wipe out the mucus in the morning and to wash the mask with soap and water or with alcohol-based disinfectant. In the morning, humidifiers should be cleaned using soap and water and dried well. Since PAP is blowing air with pressure, PAP machine will not be affected.

- Running of the machine when the patient is not wearing the mask (if for example, a patient has gone to the bathroom in the middle of the night or for some other activity) should be discouraged. One could use the auto-ON and auto-OFF function in the machines to achieve this.

- Filters should be kept clean and dry. The humidifier should be dried when not in use.

- Use of PAP should be encouraged under medical guidance.

- Discontinuation of the PAP therapy, should be done in consultation with the treating physician to explore other treatment options like positional therapy, oral devices until the quarantine period is over and the patient tests negative for COVID-19.

- It is not known whether the patient can get re-infected by using the same filters, tubing or masks. The suggestion is, if the patient can afford, to change the filters, tubing, and mask as a precautionary measure before starting to use the PAP therapy again and sleeping with family members. 
4.3.5.2 OSA-Diagnosed Patient in Hospital with COVID-19 Symptoms not Requiring Non-invasive Ventilation or Mechanical Ventilation

- Patients should be encouraged to bring a copy of their PAP device settings when they go to the hospital.

- Patients should be encouraged to bring their own PAP device and mask when they get admitted to the hospital but different hospitals have different policies regarding this.

- Masks should be cleaned daily with alcohol and hand sanitizers, though it may decrease the lifetime of the mask.

- As mentioned earlier, the present data suggest that sleep apnea does not get worse during a viral infection so PAP should be continued.

- If the patient is in respiratory distress, then the physicians' team will decide on the best therapy for the patient.

\subsubsection{Cleaning the PAP Devices and Masks at Home}

4.3.6.1 OSA Patient Without COVID-19 Diagnosis/Symptoms PAP devices and masks should be cleaned as per the manufacturer's recommendations.

\subsubsection{OSA Patient with COVID-19 Diagnosis/Symp-} toms PAP devices and masks should be cleaned with alcohol-based disinfectants.

\subsubsection{Cleaning the PAP Devices and Masks in the Sleep Laboratory}

- If in-lab titration during the COVID-19 pandemic for some emergency cases is done, then high-level disinfection in consultation with the hospital disinfection team should be done for PAP device, tubing, masks and humidifier in addition to other disinfectants in the sleep laboratory.

- If possible, the use of disposable tubing and masks during titration should be encouraged.

- PAP device filters should be changed after using them on each patient.

- While cleaning staff should take optimal precautions to prevent catching an infection.

Though extensive studies have not been done on OSA patients afflicted with COVID-19, it is believed that patients with untreated OSA are at high risk of pneumonia and respiratory infections in general. In addition to the above-mentioned guidelines, daily assessment of COVID-19 situation in the community, along with a review of the situation with local public health and state health department is advised.
This will allow informed decision making and possibly expanding the scope of the services.

\section{Conclusion}

It was an earnest attempt by the task-force of ISSR to develop guidelines for the practice of sleep medicine based on scientific evidences and other guidelines. However, information regarding COVID-19 is still evolving and that may necessitate modifications of the recommendations from time to time.

\section{References}

1. COVID-19 mitigation strategies for sleep clinics and sleep centres- reopening. https://aasm.org/covid-19-resources/covid-19mitigation-strategies-sleep-clinics-labs. Accessed 6 Jun 2020

2. Interim Infection prevention and control recommendations for patients with suspected or confirmed Coronavirus Disease 2019 (COVID-19) in healthcare settings. https://www.cdc.gov/coron avirus/2019-ncov/hcp/infection-control-recommendations.html. Accessed 6 Jun 2020

3. Altena E, Baglioni C, Espie CA, Ellis J, Gavriloff D, Holzinger B, Schlarb A, Frase L, Jernelov S, Reimann D. Dealing with sleep problems during home confinement due to the COVID-19 outbreak: practical recommendations from a task force of the European CBT-I Academy. J Sleep Res. 2020;00:e13052. https ://doi.org/10.1111/jsr.13052.

4. BSS statement on sleep related advice during coronavirus (COVID-19) pandemic. https://www.sleepsociety.org.uk/wp-conte nt/uploads/2020/04/BSS-statement-on-sleep-related-advice-durin g-the-coronavirus-Covid-19-pandemi.pdf. Accessed 6 Jun 2020

5. COVID-19 resources for clinical neurophysiology. https://www. acns.org/practice/covid-19-resources. Accessed 6 Jun 2020

6. Guidelines on preventive measures to contain spread of COVID19 in workplace settings. https://www.mohfw.gov.in/pdf/Guide linesonpreventivemeasurestocontainspreadofCOVID19inwork placesettings.pdf. Accessed 6 Jun 2020

7. COVID-19: Role of hospitals and healthcare professionals. https:// www.ima-india.org/ima/pdfdata/COVID-19-Guidelines-for-Hospi tals-n-Doctors.pdf. Accessed 6 Jun 2020

8. Besedovsky L, Lange T, Haack M. The sleep-immune crosstalk in health and disease. Physiol Rev. 2019;99:1325-80.

9. Khan IW, Juyal R, Shikha D, Gupta R. Generalized anxiety disorder but not depression is associated with insomnia: a population based study. Sleep Sci. 2018;11:166.

10. Choudhury A, Routray D, Swain S, Das AK. Prevalence and risk factors of people at-risk of obstructive sleep apnea in a rural community of Odisha, India: a community based cross-sectional study. Sleep Med. 2019;58:42-7.

11. Pazarlı AC, Ekiz T, İlik F. Coronavirus disease 2019 and obstructive sleep apnea syndrome. Sleep Breathing. 2019;2020: https:// doi.org/10.1007/s11325-020-02087-0.

12. Telemedicine practice guidelines enabling registered medical practitioners to provide healthcare using telemedicine. https:// www.mohfw.gov.in/pdf/Telemedicine.pdf. Accessed 7 Jun 2020.

13. Telemedicine-Guidelines-Gazetted-and-notified.pdf [Internet] https://www.medindia.net/indian_health_act/Telemedicine-Guide lines-Gazetted-and-notified.pdf. Accessed 7 Jun 2020 
14. Seyffert M, Lagisetty P, Landgraf J, Chopra V, Pfeiffer PN, Conte $\mathrm{ML}$, et al. Internet-delivered cognitive behavioral therapy to treat insomnia: a systematic review and meta-analysis. PLoS ONE. 2016;11:e0149139.

15. Bruyneel M. Telemedicine in the diagnosis and treatment of sleep apnoea. Eur Respir Rev. 2019;28:180093.

16. Bruyneel M. Technical developments and clinical use of telemedicine in sleep medicine. J Clin Med. 2016;5:116.

17. Singh J, Badr MS, Diebert W, Epstein L, Hwang D, Karres V, et al. American Academy of Sleep Medicine (AASM) position paper for the use of telemedicine for the diagnosis and treatment of sleep disorders. J Clin Sleep Med. 2015;11:1187-98.

18. Venkateshiah SB, Hoque R, DelRosso LM, Collop NA. Legal and regulatory aspects of sleep disorders. Sleep Med Clin. 2017;12:149-60.

19. Bornemann MA, Schenck CH. Sleep, violence, and forensic implications. Sleep disorders medicine. New York: Springer; 2017. p. $1175-1185$.

20. Krishnan V, Patil SP. Obstructive sleep apnea and transportation: medicolegal issues. Sleep Med Clin. 2013 Dec 1;8(4):591-605.

21. Gupta R, Pandi-Perumal SR, Almeneessier AS, BaHammam AS. Hypersomnolence and traffic safety. Sleep Med Clin. 2017;12(3):489-99.

22. Math SB, Manjunatha N, Naveen Kumar C, Basavarajappa C, Gangadhar BN. Telepsychiatry operational guidelines-2020. Bengaluru: 2020.

23. Kapur VK, Auckley DH, Chowdhuri S, Kuhlmann DC, Mehra R, Ramar K, Harrod CG. Clinical practice guideline for diagnostic testing for adult obstructive sleep apnea: an American Academy of Sleep Medicine clinical practice guideline. J Clin Sleep Med. 2017;13:479-504.

24. Kirk V, Baughn J, D’Andrea L, Friedman N, Galion A, Garetz S, Hassan F, Wrede J, Harrod CG, Malhotra RK. American Academy of Sleep Medicine position paper for the use of a home sleep apnea test for the diagnosis of OSA in children. J Clin Sleep Med. 2017 Oct 15;13(10):1199-203.

25. Enabling delivery of essential health services during COVID-19 outbreak: guidance note. https://www.mohfw.gov.in/pdf/Essen tialservicesduringCOVID19updated0411201.pdf. Accessed 7 Jun 2020

26. The Epidemic Diseases (Amendment) Ordinance, 2020. https:// egazette.nic.in/WriteReadData/2020/219108.pdf. Accessed 7 Jun 2020

27. Nigro CA, Borsini E, Dibur E, Larrateguy L, Cazaux A, Elias C, et al. Indication of CPAP without a sleep study in patients with high pretest probability of obstructive sleep apnea. Sleep Breath. 2019. https://doi.org/10.1007/s11325-019-01949-6.

28. Ferioli M, Cisternino C, Leo V, Pisani L, Palange P, Nava S. Protecting healthcare workers from SARS-CoV-2 infection: practical indications. Eur Respir Rev. 2020;29:200068. https://doi. org/10.1183/16000617.0068-2020.

Publisher's Note Springer Nature remains neutral with regard to jurisdictional claims in published maps and institutional affiliations.

\section{Affiliations}

\section{Ravi Gupta ${ }^{1}$. V. Mohan Kumar ${ }^{2} \cdot$ Manjari Tripathi $^{3} \cdot$ Karuna Datta $^{4} \cdot$ Manjunatha Narayana $^{5}$. Kripesh Ranjan Sarmah ${ }^{6} \cdot$ Manvir Bhatia $^{7} \cdot$ Preeti Devnani $^{8} \cdot$ Sourav Das $^{9} \cdot$ Deepak Shrivastava $^{10}$. Rama Devi Gourineni ${ }^{11,12} \cdot$ Tripat Deep Singh $^{13} \cdot$ Apar Jindal $^{14} \cdot$ Hruda Nanda Mallick $^{2}$}

Hruda Nanda Mallick

drhmallick@yahoo.com

1 Department of Psychiatry, All India Institute of Medical Sciences, Rishikesh 249203, India

2 Department of Physiology, All India Institute of Medical Sciences, New Delhi 110029, India

3 Department of Neurology, All India Institute of Medical Sciences, New Delhi, India

4 Department of Sports Medicine, Armed Forces Medical College, Pune, India

5 Department of Psychiatry, National Institute of Mental Health and Neurosciences, Bengaluru, India

6 Apollo Hospital, Guwahati, India

7 Neurology and Sleep Centre, New Delhi, India

8 Cleveland Clinic, Abu Dhabi, UAE
$9 \quad$ Somnos Sleep Clinic, Kolkata, India

10 Division of Pulmonary, Critical Care and Sleep, Department of Medicine and Medical Director, SJGH Sleep Center, UC Davis School of Medicine, Sacramento, CA, USA

11 Neurology, Northwestern Feinberg School of Medicine, Chicago, USA

12 Sleep Department, Amara Hospital, Tirupati, India

13 Sleep- Asia-Pacific, Philips Electronics, Singapore, Singapore

14 Institute of Heart and Lung Transplant, Gleneagles Global Health City, Cheran Nagar, Perumbakkam, Chennai 600 100, India 